\title{
An Optimal Execution Time Estimate of Static versus Dynamic Allocation in Multiprocessor Systems
}

\author{
Håkan Lennerstad Lars Lundberg
}

January 30, 1996

\section{Abstract}

Consider a multiprocessor with $k$ processors.

Assume we are running programs consisting of $n$ processes, $n>k$. Let $T_{s}(P)$ and $T_{d}(P)$ denote the execution times for the program $P$ with optimal static and dynamic allocations respectively.

We derive a general and explicit formula for the optimal execution time ratio $g(n, k)=\max T_{s}(P) / T_{d}(P)$, where the maximum is taken over all programs $P$ consisting of $n$ processes.

The cost of transferring processes between processors in the dynamic case, and the execution time of synchronizing signals are neglected only.

Basic properties of the function $g(n, k)$ are established, from which we obtain a global description of the function. A plot of $g(n, k)$ is included. It is a perhaps surprising fact that for $n, k \leq 100$, the values of $g(n, k)$ does not exeed 3. However, $g(n, k)$ is unbounded.

The results are obtained by investigating a mathematical formulation. The mathematical tools involved are essentially tools of elementary combinatorics. The formula is a combinatorial function applied on certain extremal matrices corresponding to extremal programs. It is mathematically complicated but rapidly computed for reasonable $n$ and $k$, in contrast to the np-completeness of the problems of finding optimal allocations. 


\section{Introduction}

An optimal bound of the efficiency of dynamic compared to static parallel computing can be obtained by solving a mathematical formulation as follows.

Consider a multiprocessor with $k$ processors. We calculate the quotient

$$
g(n, k)=\max _{P} \frac{T_{s}(P)}{T_{d}(P)}
$$

where the maximum is taken over all programs $P$ with $n$ processes. $T_{s}(P)$ and $T_{d}(P)$ are the optimal execution times with static and dynamic allocations, respectively. That is, we calculate the optimal worst case estimate.

In this context, the only difference between static and dynamic allocation is that in the dynamic case processes, after having been started, can be transferred to another processor. The cost of this transferrence is neglected. In the static case processes are always processed to the end on the processor on which it was initialized. If a process is put into a waiting state, it will later be restarted on the same processor.

A synchronizing signal is a command in a process which activates or deactivates another process. The execution time of the synchronizing signals are neglected. A program $P$ consists of $n$ processes, of possibly very different execution times, containing synchronizing signals, possibly defining a very complicated structure of the program $P$.

Consider such a program $P$, and assume that we have found an optimal dynamic allocation, with execution time $T_{d}(P)$. Next we introduce a discretization of the time interval in subintervals $\left(t_{i}, t_{i+1}\right)$ of equal length, such that all synchronizing signals and process terminations appear on the time points $t_{i}$, where $t_{i}=\frac{i}{m} T_{d}(P), i=0, \ldots, m$. It is convenient to assume further that $\left(\begin{array}{l}n \\ k\end{array}\right)$ divides $m$. Such a discretization is possible if all synchronizing signals and process terminations occur at rational time points, which we can assume. Observe that $m$ might be very large even if the program $P$ is small and has a simple structure. However, $m$ plays no important role in the theory.

Consequently, during a time interval $\left(t_{i}, t_{i+1}\right)$, no process starts, and no process stops.

From the program $P$ we now construct another program $P^{\prime}$ by introducing new synchronizing signals and prolonging certain processes. At all time points $t_{i}$ we introduce all possible synchronizing signals between the processes. Thus, in the program $P^{\prime}$, all processes in the interval $\left(t_{i-1}, t_{i}\right)$ have to be completed before any parts of the processes corresponding to the 
interval $\left(t_{i}, t_{i+1}\right)$ can be started. In the program $P^{\prime}$ all processors are busy at all time intervals. This is achieved by, if necessary, prolonging certain processes in $P$ so that $T_{d}(P)$ is unchanged.

Both these changes may increase the static execution time, so we have $T_{s}(P) \leq T_{s}\left(P^{\prime}\right)$. Clearly, $T_{d}(P)=T_{d}\left(P^{\prime}\right)$. Hence,

$$
\frac{T_{s}(P)}{T_{d}(P)} \leq \frac{T_{s}\left(P^{\prime}\right)}{T_{d}\left(P^{\prime}\right)}
$$

But, since the programs $P^{\prime}$ consistute a subset of the programs $P$ we consider, we actually have

$$
\max _{P} \frac{T_{s}(P)}{T_{d}(P)}=\max _{P} \frac{T_{s}\left(P^{\prime}\right)}{T_{d}\left(P^{\prime}\right)}
$$

We next represent a program $P^{\prime}$ by a $m \times n$ matrix of 0 :s and 1 :s only. Here each process is represented by a column, and each time period is represented by a row. The entry at the position $i, j$ is 1 if the $j$ :th process is active between $t_{i-1}$ and $t_{i}$. The entry is 0 if the $j$ :th process is inactive during this interval. Each row contains exactly $k 1: \mathrm{s}$, since each processor is constantly busy, and we have $k$ processors. In the sequel such a matrix is referred to as an $m, n, k$-type matrix. The main part of the report analyzes these matrices. We for example characterize the type of matrix which corresponds to the worst case.

What is the optimal static execution time of the program $P^{\prime}$ ? To compute this we need to decide how the $n$ processes are to be allocated to the $k$ processors. Since every process in the static case is to be executed on one processor only, the static allocation corresponds to a way of grouping the $n$ columns of the matrix together in $k$ sets, one set for each processor. Because of the complete synchronization, at each step the processors have to wait for the slowest processor. This is the processor which has the highest number of processes to execute, i.e. the maximum number of 1 :s in one group. Clearly, the static execution time is the sum of these waiting times, muliplied by the factor $T_{d}(P) / m$.

Thus, by summing the vectors in each group, vectors denoting the time needed for each processor at each time interval are calculated. By taking maxima of these vectors, we derive the static execution time at each time interval. The component sum of the resulting vector is the static execution time. This is the optimal static execution time $T_{s}\left(P^{\prime}\right)$ if we have found the best allocation, i.e. a way of grouping the $n$ columns together in $k$ sets 
which minimizes the static execution time. In the following we denote

$$
T(P)=T_{s}(P) \frac{m}{T_{d}(P)},
$$

i.e. we compute the time in the unit $\frac{T_{d}(P)}{m}$. The optimal dynamic execution time in this unit is $m$.

In the main result we find the function $g$ representing the worst case, i.e. for any program $P_{0}$ :

$$
\frac{T_{s}\left(P_{0}\right)}{T_{d}\left(P_{0}\right)} \leq g(n, k)=\max _{P} \frac{T_{s}(P)}{T_{d}(P)}=\max _{P} \frac{T(P)}{m} .
$$

For a full presentation of how the problem appears in parallel computing and for the significance of the results we refer to Comparing the Performance of Optimal Dynamic and Static Process Allocation (L. Lundberg and H. Lennerstad, Research Report, University of Karlskrona/Ronneby, Sweden).

In the next section we formulate the mathematical problem.

In section 4 the basic formula for the function $g(n, k)$ is stated and proved.

Section 5 contains a plot of $g(n, k)$ and results from which a global description of the function $g(n, k)$ follows.

There are theoretical results on so called 0,1-matrices: matrices where each entry is 0 or 1 . However non of these appear to be applicable to our formulation. The concluding reference list consists of different approaches in the problem of comparing static and dynamic allocation in multiprocessor systems, as well as results on 0,1-matrices.

\section{The mathematical formulation}

As described in the introduction, the corresponding mathematical problem can be formulated as follows:

Consider an $m \times n$ matrix $P$ of 0 :s and 1:s only, such that each row has exactly $k 1: \mathrm{s}$, and thus $n-k 0: \mathrm{s}, 1 \leq k \leq n$. These matrices are referred to as $m, n, k$-type matrices.

The column vectors of $P$ will sometimes be denoted by $v_{i}$. Consider a partition $A$ of the $n$ vectors $v_{i}$ into $k$ sets. Observe that the number of sets equals the number of $1: \mathrm{s}$ on each row in $P$. We will be mostly concerned with partitions where the sizes of the sets in the partition differ as little as possible. If $n / k$ is an integer $w$ this means that every set has $w$ members. 
Denote the integer part of $n / k$, the floor function, by $\lfloor n / k\rfloor$, and the smallest integer greater than or equal to $n / k$, the ceiling function, by $\lceil n / k\rceil$. If $n / k$ is not an integer, the sets in a partition where the sizes differ as little as possible have $\lfloor n / k\rfloor$ or $\lceil n / k\rceil$ members.

Given any partition $A$ of the column vectors in $k$ sets, we form a quantity $T_{A}(P)$ as follows.

The vectors in each group are added together, making $k$ vectors of nonnegative integers from the $n$ column vectors. Then by taking the maximum of these vectors, componentwise, we obtain one single vector of positive integers. All vectors are of course $m$-dimensional. The sum of the components of the final vector is the quantity $T_{A}(P)$. In formula:

$$
T_{A}(P)=\sum_{j=1}^{m} \max _{l=1, \ldots, k}\left(\sum v_{i}\right),
$$

where the last sum is taken over the indexes $i$ which belongs to the $l$ :th partition set.

We want to choose the partition $A$ so that $T_{A}(P)$ is minimal. The interesting quantity is thus

$$
T(P)=\min _{\text {all partitions } A} T_{A}(P) .
$$

The function $g(n, k)$ is defined by

$$
g(n, k)=\max \left\{\frac{T(P)}{m} \text {, all } m, n, k-\text { type matrices } P\right\} .
$$

For given $m, n$ and $k$, we will thus be concerned with the problem of calculating $\max T(P)$, over all $m, n, k$-type matrices $P$.

A natural conjencture is the estimate $T(P) / m \leq g(n, k) \leq 2$. In the case $n \leq 2 k$ it is immediately seen to be true, by simply grouping the column vectors together in pairs. The conjencture is not true in the general case, as one can see in the plot. However, for a partition where the size of the sets differ as little as possible, the largest set has $\lceil n / k\rceil$. Then the maximum number of $1: \mathrm{s}$ in a set is $\min (\lceil n / k\rceil, k)$ : both the number of slots in the largest set and the total number of 1:s provide bounds. We thus obtain a crude estimate $g(n, k) \leq \min (\lceil n / k\rceil, k)$. We will frequently compare the optimal estimate $g(n, k)$ with this crude estimate. 
Let us now, before the main result, introduce and summarize the notation which is relevant in this situation.

We say that a matrix $A$ is of $\boldsymbol{m}, \boldsymbol{n}, \boldsymbol{k}$-type if it has $m$ rows and $n$ columns, all entries are $0: \mathrm{s}$ or $1: \mathrm{s}$, and each row has exactly $k 1: \mathrm{s}$, where $1 \leq k \leq n$.

We call a matrix $P$ balanced if all possible rows, that is, if all $\left(\begin{array}{l}n \\ k\end{array}\right)$ permutations of the $k 1$ :s, occur equally frequently as rows of $P$. The number of rows is thus necessarily divisible by $\left(\begin{array}{l}n \\ k\end{array}\right)$.

We also need the following three combinatorial functions. Let $I$ be a finite sequence of nonnegative integers. Then we define:

$b(I)=$ the number of distinct integers in $I$

$a(I, j)=$ the number of occurencies of the $j$ :th distinct integer in $I$, enumerated in size order, $1 \leq j \leq b(I)$.

$\pi(k, w, q, l)=$ the number of permutations of $q 1$ :s distributed in $k w$ slots, which are divided in $k$ sets with $w$ slots in each, such that the set with maximum number of $1: s$ has exactly $l$ 1:s. 


\section{The optimal estimate}

With this notation, the main result is contained in the following theorem.

THEOREM 1 Given positive integers $m, n$ and $k$.

In the case where $w=n / k$ is an integer, we have for all matrices $P$ of $m, n, k$-type:

$$
T(P) / m \leq g(n, k)=\frac{1}{\left(\begin{array}{l}
n \\
k
\end{array}\right)} \sum_{l=1}^{\min (w, k)} l \pi(k, w, k, l) .
$$

If $w=n / k$ is not an integer, we let $w=\lfloor n / k\rfloor$ and denote the remainder of $n$ divided by $k$ by $n_{k}$, i.e. $n_{k}=n-k\lfloor n / k\rfloor$. Then we have for all matrices $P$ of $m, n, k$-type:

$$
\begin{gathered}
T(P) / m \leq g(n, k)=\frac{1}{\left(\begin{array}{l}
n \\
k
\end{array}\right)} \sum_{l_{1}=\max \left(0,\left\lceil\frac{k-\left(k-n_{k}\right) w}{n_{k}}\right\rceil\right)}^{\min (w+1, k)} \sum_{l_{2}=\max \left(0,\left\lceil\frac{k-l_{1} n_{k}}{k-n_{k}}\right\rceil\right)}^{\min \left(w, k-l_{1}\right)} \max \left(l_{1}, l_{2}\right)( \\
\left.\sum_{i=\max \left(l_{1}, k-l_{2}\left(k-n_{k}\right)\right)}^{\min \left(l_{1} n_{k}, k-l_{2}\right)} \pi\left(n_{k}, w+1, i, l_{1}\right) \pi\left(k-n_{k}, w, k-i, l_{2}\right)\right) .
\end{gathered}
$$

The function $\pi(k, w, q, l)$ is 0 if $\min (q, w)<l$ or if $q>k l$, otherwise it is given by

$\pi(k, w, q, l)=\left(\begin{array}{c}w \\ l\end{array}\right) \sum_{I}\left(\begin{array}{c}w \\ i_{1}\end{array}\right) \cdot \ldots \cdot\left(\begin{array}{c}w \\ i_{k-1}\end{array}\right) \frac{k !}{\Pi_{j=1}^{b\left(\left\{l, i_{1}, \ldots, i_{k-1}\right\}\right)} a\left(\left\{l, i_{1}, \ldots, i_{k-1}\right\}, j\right) !}$.

Here the sum is taken over all sequences of nonnegative integers $I=$ $\left\{i_{1}, \ldots, i_{k-1}\right\}$ which are decreasing: $i_{j} \geq i_{j+1}$ for all $j=1, \ldots, k-2$, bounded by $l: i_{1} \leq l$, and have sum $q-l: \sum_{j=1}^{k-1} i_{j}=q-l$.

For each $m, n, k$-type matrix $P$ the minimum

$$
T(P)=\min _{\text {all partitions } A} T_{A}(P)
$$

is attained for a partition where the sizes of the sets in the partition differ as little as possible.

The bound is optimal in the sense that if $\left(\begin{array}{l}n \\ k\end{array}\right)$ divides $m$, in which case there exist balanced matrices, we have $T(P) / m=g(n, k)$ for all balanced matrices $P$. 
The sequences in the function $\pi$ can easily be generated by the algorithm described in the following lemma. We say that the least decreasing sequence of length $\mu$ and sum $\sigma$ is the sequence $\left\{\left\lceil\frac{\sigma}{\mu}\right\rceil, \ldots,\left\lceil\frac{\sigma}{\mu}\right\rceil,\left\lfloor\frac{\sigma}{\mu}\right\rfloor, \ldots,\left\lfloor\frac{\sigma}{\mu}\right\rfloor\right\}$. If $\sigma_{\mu}$ is the remainder when $\sigma$ is divided by $\mu$, the number of $\left\lceil\frac{\sigma}{\mu}\right\rceil: \mathrm{s}$ is $\sigma_{\mu}$, and the number of $\left\lfloor\frac{\sigma}{\mu}\right\rfloor$ :s is $\mu-\sigma_{\mu}$, making the sum of the sequence $\sigma$.

LEMMA 2 Let $\lambda$ and $\sigma$ be nonnegative integers and $\mu$ be a positive integer such that $\lambda \leq \sigma \leq \lambda \mu$.

Every sequence of $\mu$ integers in the interval $0 \leq i \leq \lambda$ which is decreasing, bounded by $\lambda$ and has sum $\sigma$ is generated exactly once by the following algorithm:

1. Take $I$ as the least decreasing sequence of length $\mu$ and sum $\sigma$.

2. Find the rightmost position in I, say $j_{1}$, which fullfills:

(a) $i_{j_{1}}<l$

(b) $i_{j_{1}}<i_{j_{1}-1}$ or $j_{1}=1$

(c) $i_{j_{1}+1}>0$

The algorithm terminates if no such $j_{1}$ can be found.

3. The next sequence is obtained from I by increasing the entry in position $j_{1}$ by one, and replacing the subsequence $\left\{i_{j_{1}+1}, \ldots, i_{\mu}\right\}$ with the least decreasing subsequence of length $\mu-j_{1}$ and sum $\sum_{j=j_{1}+1}^{\mu} i_{j}-1$.

4. Go to step 2.

Proof of the lemma: It is immediately clear that the starting sequence is decreasing, has sum $\sigma$ and has entries in the interval $0 \leq i \leq l$. It is also obvious that these properties are preserved by the algorithm.

Consider a sequence $I$ of this kind. If it is not the last one generated by the algorithm, the next sequence will have its entry at $j_{1}$ increased. This entry will not decrease again unless an entry to the left is increased. By reapplying this argument we find that no sequence is generated twice by the algorithm.

What is left to prove is that the algorithm generates all such sequences. This is done by induction over the length $\mu$ of the sequence.

Assume that this is true for all decreasing sequences of length $\mu$.

For $\mu=1$ the only sequence is $\{\sigma\}$, which is the starting sequence of the algorithm and the only sequence generated by the algorithm. 
We want to prove that the algorithm generates all decreasing sequences of length $\mu+1$, sum $\sigma$ and bound $\lambda$.

These sequences are $\left\{\min (\lambda, \sigma), I_{1}\right\}, \ldots,\left\{\lceil\sigma / \mu\rceil, I_{j_{0}}\right\}$, where $I_{1}, \ldots, I_{j_{0}}$ are decreasing sequences of length $\mu$, bounded by $\min (\lambda, \sigma), \ldots,\lceil\sigma / \mu\rceil$ and with sums $\sigma-\min (\lambda, \sigma), \ldots, \sigma-\lceil\sigma / \mu\rceil$ respectively. Now the algorithm with length $\mu+1$ applied on the sequences $\left\{\min (\lambda, \sigma), I_{1}\right\}, \ldots,\left\{\lceil\sigma / \mu\rceil, I_{j_{0}}\right\}$ with the bounds $\min (\lambda, \sigma), \ldots,\lceil\sigma / \mu\rceil$ is, except for the first entry, the same as the algorithm with length $\mu$ applied on the sequences $\left\{I_{1}, \ldots, I_{j_{0}}\right\}$ with the bounds $\min (\lambda, \sigma), \ldots,\lceil\sigma / \mu\rceil$. By the induction assumption this generate all decreasing sequences of length $\mu$. The lemma is proved.

Proof of the theorem: Consider an arbitrary matrix $P$ of $m, n, k$-type. Let $A$ be a partition where the sizes of the sets differ as little as possible. We will later prove that the minimum is attained for such a partition. Observe that this is equivalent to $s_{\max }-s_{\min } \leq 1$, if $s_{\max }$ is the number of vectors in a set with most vectors, and $s_{\min }$ is the number of vectors in a set with fewest vectors.

Each row in $P$ can be regarded as a permutation of $k 1$ :s in $n$ slots. There are of course $\left(\begin{array}{l}n \\ k\end{array}\right)$ such. Further there exist $n$ ! permutations of the columns of $P$, where each permutation produces a possibly different $m, n, k$-type matrix $P_{i}, i=1, \ldots, n$ !. When we permute the column vectors we permute the rows, all possible rows appear if we perform all possible column permutations. We can also regard these permutations of the columns as different partitions $A_{i}$ of the original matrix $P$. Next we construct a matrix $P^{*}$ from the matrices $P_{i}$, which has $m n$ ! rows and $n$ columns, using the following duplication argument. This will provide control of the partitions.

The first $m$ rows of $P^{*}$ consistute the matrix $P$ itself. The next $m$ rows constitute the matrix $P$ where the columns are permuted according to a specific permutation, which is not the identity. The following $n !-2$ blocks of $m$ rows contain all other permutations of the columns of the matrix $P$. We know three facts about the matrix $P^{*}$ which makes this procedure useful:

1. Every row in $P^{*}$ occur exactly as many times as any other different row in the matrix $P^{*}$. Every possible row does appear. That is, $P^{*}$ is a balanced matrix.

2. Each column permutation represents a partition $A_{i}$ of the columns of $P$, so that $T_{A}\left(P_{i}\right)=T_{A_{i}}(P), i=1, \ldots, n !$ 
3. The quantities $T\left(P_{i}\right), i=1, \ldots, k$ ! relate to the quantity $T\left(P^{*}\right)$ as $T\left(P^{*}\right)=\sum_{i=1}^{n !} T\left(P_{i}\right)$.

Thus, since $T(P)$ arises from the partition $A_{i}$ which gives the smallest value of $T_{A_{i}}(P)=T_{A}\left(P_{i}\right)$, we have $T(P) \leq T_{A_{i}}(P)=T_{A}\left(P_{i}\right)$ for all $i=$ $1, \ldots, n$ !. We then obtain from (3) above:

$$
T(P) \leq \sum_{i=1}^{n !} T\left(P_{i}\right) / n !=T\left(P^{*}\right) / n ! .
$$

We have thus established that the balanced matrices represent the worst case.

A balanced matrix has a particularly simple structure. Each permutation in the matrix $P^{*}$ is repeated $m$ times if we count every 1 and every 0 as distinct. By releasing this distinction, each permutation really is repeated $m n !(n-k)$ ! times. Since this factor only multiplies all occuring numbers, what is left to study is the balanced matrix $\tilde{P}$ where each permutation occurs exactly once. We have:

$$
\begin{aligned}
T\left(P^{*}\right) & =m n !(n-k) ! T(\tilde{P}), \text { so } \\
T(P) \leq T\left(P^{*}\right) / n ! & =m n !(n-k) ! T(\tilde{P}) / n !=\frac{m}{\left(\begin{array}{c}
n \\
k
\end{array}\right)} T(\tilde{P}) .
\end{aligned}
$$

The matrix $\tilde{P}$ has $\left(\begin{array}{l}n \\ k\end{array}\right)$ rows and $n$ columns, and contains each permutation of the $k 1: \mathrm{s}$ in the $n$ slots exactly once. The columns are grouped together in sets with $\lceil n / k\rceil$ or $\lfloor n / k\rfloor$ members. For the maximum operation we are interested in the number of $1: \mathrm{s}$ in the set with most $1: \mathrm{s}$, we here have say $l 1:$ s. For the component sum of the final vector we are interested in the number of permutations where we have exactly $l 1$ :s in the set of maximum $1: \mathrm{s}$.

Consider first the case when $n / k=w$ is an integer. Then all sets in the partition contains $w$ vectors.

If we for simplicity denote the number of permutations giving $l$ by $\pi(l)$, we have $T(\tilde{P})=\sum_{l=1}^{\min (w, k)} l \pi(l)$.

For the sake of clarity, we compute a few special cases of $\pi(l)$ before considering the general situation.

In the following special cases we assume that $k \leq w$.

1. Clearly, $\pi(1)=w^{k}$, since here we have to put exactly one 1 in each set. In the first set the 1 can be put in $w$ different slots, as it can in all other $k$ sets. 
2. $\pi(k)=\left(\begin{array}{c}w \\ k\end{array}\right) k$. Here the first factor comes from the ways of putting all the $1: \mathrm{s}$ in one set, and the factor $k$ is the number of sets where this can be done.

3. $\pi(k-1)=\left(\begin{array}{c}w \\ k-1\end{array}\right) w\left(\begin{array}{l}k \\ 2\end{array}\right)$. The second factor in this case is the number of slots to put the single 1 . The last factor is the number of ways to distribute the two different sets among the $k$.

4. $\pi(k-2)=\left(\begin{array}{c}w \\ k-2\end{array}\right)\left(\left(\begin{array}{l}w \\ 2\end{array}\right)\left(\begin{array}{l}k \\ 2\end{array}\right)+w\left(\begin{array}{l}k \\ 3\end{array}\right) / 2\right)$. The first term is the number of ways to produce $l=k-2$ with the distribution $k-2,2,0, \ldots, 0$ of the $1: \mathrm{s}$ in the $k$ sets. The other term arises from the distribution $k-2,1,1,0, \ldots, 0$.

Generally, to start with we have a number of ways to distribute $k 1$ :s in $k$ sets regardless of order both in each set and between the sets. These ways are represented by the decreasing sequences. The order in each set is here disregarded in such a way that only the number of $1: \mathrm{s}$ is significant. The order between the sets is disregarded by chosing one specific order: decreasing sequences.

In a set of $i 1$ :s and $w-i 0$ :s there are $\left(\begin{array}{c}w \\ i\end{array}\right)$ different permutations. So, taking the order in the sets into account, if we have $\left\{i_{1}, \ldots, i_{k}\right\} 1$ :s in the $k$ sets respectively, there are $\left(\begin{array}{c}w \\ i_{1}\end{array}\right) \cdot \ldots \cdot\left(\begin{array}{c}w \\ i_{k}\end{array}\right)$ different permutations. Since the maximum number $l$ must appear, we always get a factor $\left(\begin{array}{c}w \\ l\end{array}\right)$, which can be factored out. The remaining sequence is of length $k-1$ and has $\operatorname{sum} k-l$.

We next consider the order between the sets. There are $k$ ! permutations of the sets. If there are $a\left(\left\{l, i_{1}, \ldots, i_{k-1}\right\}, j\right)$ ! sets with $i_{j} 1$ :s, we get no new permutations by permuting within this group of sets. Hence the factor from permuting the sets is

$$
\frac{k !}{\Pi_{j=1}^{b\left(\left\{l, i_{1}, \ldots, i_{k-1}\right\}\right)} a\left(\left\{l, i_{1}, \ldots, i_{k-1}\right\}, j\right) !} .
$$

The number of permutations which give the number $l$ is thus

$$
\pi(l)=\left(\begin{array}{c}
w \\
l
\end{array}\right) \sum_{I}\left(\begin{array}{c}
w \\
i_{1}
\end{array}\right) \cdot \ldots \cdot\left(\begin{array}{c}
w \\
i_{k-1}
\end{array}\right) \frac{k !}{\Pi_{j=1}^{b\left(\left\{l, i_{1}, \ldots, i_{k-1}\right\}\right)} a\left(\left\{l, i_{1}, \ldots, i_{k-1}\right\}, j\right) !},
$$

summing over the decreasing sequences of length $k-1$, sum $k-l$ and bound $l$. 
In the case when $n / k$ is not an integer, we work with a partition where the $n_{k}$ leftmost sets have $w+1=\lceil n / k\rceil$ vectors and the rightmost $k-n_{k}$ have $w=\lfloor n / k\rfloor$ vectors.

The formula in this case is derived from the previous formula by introducing the possibility that the number of sets, $k$, and the number of $1: \mathrm{s}$, $q$, are not equal. By summing over all possible maximums to the left $\left(l_{1}\right)$ and to the right $\left(l_{2}\right)$, and over all possible numbers of 1 :s to the left $(i)$, the results for general $n$ and $k$ follow. The bounds of the indexes appear from the limitations of the number of 1 :s which there is room for to the left or to the right in the different cases, and from the minimum number of $1: \mathrm{s}$ according to $l_{1}, l_{2}$ and $i$.

We finally prove the optimality result.

If the matrix $P$ is balanced with $\left(\begin{array}{l}n \\ k\end{array}\right)$ rows, then $P=\tilde{P}$, and the above calculation is true with equality if $\min _{\text {all partitions } A} T_{A}(P)$ is attained for a partition where the sizes of the sets differ as little as possible. We next prove that this is so for balanced matrices. Thus the bound is attained for programs corresponding to balanced matrices.

Let $A$ be a partition of the $n$ columns into $k$ sets, with $\left\{i_{1}, \ldots, i_{k}\right\}$ columns in each set, respectively. Assume that there are $i_{j}: \mathrm{s}$, say $i_{1}$ and $i_{2}$, such that $i_{1} \geq i_{2}+2$, otherwise we have the above mentioned type of partition. From the partition $A$ we will obtain a new partition $A^{\prime}$ by transferring the $i_{1}$ :th vector from the first set to the second. We show that the result is never worse for this partition, i.e. $T_{A^{\prime}}(P) \leq T_{A}(P)$. By repeating this transformation, a partition where the sizes of the sets differ as little as possible is finally derived, and the result follows from the inequality.

Consider a row in $\tilde{P}$, that is a permutation $p=\{p(i)\}_{i=1}^{n}$ of 1 :s and 0:s. If $p\left(i_{1}\right)=0$ nothing happens on this row. If $p\left(i_{1}\right)=1$ and $\sum_{i=1}^{i_{1}} \leq \sum_{i=i_{1}+1}^{i_{2}}$, the maximum taken over this row does increase, unless other sets contain more 1:s. These are the critical permutations. However, for each such permutation there is a unique permutation $\tilde{p}$ where the row maximum in such a case will decrease. $\tilde{p}$ is defined as a partial mirror image:

$$
\tilde{p}(i)= \begin{cases}p\left(i_{1}+i_{2}+1-i\right) & \text { if } i=1, \ldots, i_{2} \text { or } i=i_{1}+1, \ldots, i_{1}+i_{2} \\ p(i) & \text { all other } i\end{cases}
$$

Since $\sum_{i=1}^{i_{2}} p(i)<\sum_{i=i_{1}+1}^{i_{1}+i_{2}} p(i)$ for a critical permutation, we know that $\tilde{p}$ is not critical if $p$ is. Further it follows from $\tilde{\tilde{p}}=p$ that $\sim$ is a bijection. Hence, every critical permutation $p$ can be paired with a unique permutation $\tilde{p}$, since $\tilde{P}$ contains each permutation exactly once. 
Because of $p(i)=\tilde{p}(i)$ for $i=i_{1}+i_{2}+1, \ldots, n$ and $\sum_{i=1}^{i_{1}} \tilde{p}(i) \geq \sum_{i=i_{1}+1}^{i_{2}} p(i)$, it is clear that if the partition change causes the maximum on the row with $p$ to increase, the maximum on the row with $\tilde{p}$ will certainly decrease. The proof of the theorem is completed.

Given $n$ and $k$, how many matrices of $\left(\begin{array}{l}n \\ k\end{array}\right), n, k$-type are balanced? Since we obtain all balanced matrices by permuting the rows in a given balanced matrix, there are $\left(\begin{array}{l}n \\ k\end{array}\right)$ ! balanced matrices. There are in total $\left(\begin{array}{l}n \\ k\end{array}\right)\left(\begin{array}{l}n \\ k\end{array}\right)$ different matrices of $\left(\begin{array}{l}n \\ k\end{array}\right), n, k$-type: for each row there are $\left(\begin{array}{l}n \\ k\end{array}\right)$ possibilities and we have $\left(\begin{array}{l}n \\ k\end{array}\right)$ rows. The relative number of balanced matrices can now be estimated by Stirlings formula $n ! \approx\left(\frac{n}{e}\right)^{n} \sqrt{2 \pi n}$ :

$$
\frac{\left(\begin{array}{l}
n \\
k
\end{array}\right) !}{\left(\begin{array}{l}
n \\
k
\end{array}\right)\left(\begin{array}{l}
n \\
k
\end{array}\right)} \approx\left(\frac{1}{e}\right)\left(\begin{array}{l}
n \\
k
\end{array}\right) \sqrt{2 \pi\left(\begin{array}{l}
n \\
k
\end{array}\right)},
$$

which tend to zero rapidly as $\left(\begin{array}{l}n \\ k\end{array}\right) \rightarrow \infty$. The significance of this is limited, however, since it is clear that for increasing $n$ and $k$, matrices very close to balanced matrices play a role increasingly similar to the role of the balanced ones.

\section{Properties of the function $g(n, k)$}

The function $g(n, k)$ can be regarded as a weighted mean value of the integers $l=1,2, \ldots, \min (\lceil n / k\rceil, k)$, where the weights are the number of permutations which gives $l$ to the final sum, divided by the total number of permutations $\left(\begin{array}{l}n \\ k\end{array}\right)$. This fact is exploited several times in this section. The crude estimate can be viewed as the estimate of this weighted mean value by the largest integer $\min (\lceil n / k\rceil, k)$.

From the following theorem we will be able to derive the following description of $g(n, k)$ for large $n$ and $k$. We find that $g(n, k)$ like the crude estimate have an infinite series of plateaus: for each positive integer $w$ there is a planar or almost planar unbounded part which is a subset of the domain $n>(w-1) k, n<w k$ where the values are close to $w$. After the proof of the theorem this property is given a more precise formulation. In the included plot the first two plateaus, $g=1$ and $g=2$ can be seen. It seems like the distance from the origin to the plateau $g=z$ increases very rapidly with $z$. The plateau $g=3$, for example, appear beyond $n=100$.

The crude and the optimal estimates often quantitatively differ strongly. One simple example of this is the fact that for $1 \leq n \leq 100,1 \leq k \leq 100$, 
the maximum of the crude estimate is 10 while the maximum of $g$ is 2.8 . In particular when $n>>k$, the crude estimate seems to deviate strongly from the optimal.

THEOREM 3 The function $g(n, k)$ has the following properties:

1. $g(n, k)$ is increasing as a function of $n$.

2. For any positive integer $w, g(w k, k)$ is increasing as a function of $k$.

3. For all positive integers $\nu$ and $w$ we have:

$$
\begin{array}{r}
w+1-\left(1-w^{-(w+1)}\right)^{\nu} \geq \lim _{k \rightarrow \infty} g(w k+\nu, k) \geq \\
\max \left(w, w+1-w\left(1-w^{-(w+1)}\right)^{\nu}\right) .
\end{array}
$$

4. The function $g(n, k)$ is unbounded.

Proof: (1): $g(n, k)=\min _{\text {all partitions } A} T_{A}(P) / m$, where $P$ is a balanced $m, n, k$-type matrix. Let $A$ be a partition where the sizes of the sets differ as little as possible. Now, by adding to the matrix $P$ one column of only zeros we obtain the non-balanced $m, n+1, k$-type matrix $P_{0}$. This column is added to the partition $A$, producing $A_{0}$, in such a way that also $A_{0}$ have sizes of the sets which differ as little as possible. By the proof of theorem 2 it follows that $g(n, k)=T_{A}(P) / m=T_{A_{0}}\left(P_{0}\right) / m \leq T_{A_{0}}\left(\tilde{P}_{0}\right) / m=g(n+1, k)$, where $\tilde{P}_{0}$ is a balanced matrix of $m, n+1, k$-type. We can choose $m$ so that it is divisible by both $\left(\begin{array}{l}n \\ k\end{array}\right)$ and $\left(\begin{array}{c}n+1 \\ k\end{array}\right)$.

(2): The increasing property in (2) follows by adding $w$ columns to the matrix $P$, with an argument similar to the one in the proof of (1).

(3): Assume that $k$ is large, so that $k \leq(w+1) \nu$. When attempting to estimate $g(w k+\nu, k)$ we of course consider $m, w k+\nu, k$-type matrices, so we have $\nu$ sets with $w+1$ column vectors, and $k-\nu$ sets with $w$ vectors. The limit will be derived by estimating the weight to $w+1$.

How many of the total $\left(\begin{array}{c}w k+\nu \\ k\end{array}\right)$ permutations give rise to $w+1$ ? To begin with, $\left(\begin{array}{c}w k+\nu-(w+1) \\ k-(w+1)\end{array}\right)$ of the permutations have all 1:s in the first set. There are $\nu$ sets with size $w+1$, so we have $\nu$ sets of permutations with at least one set with all 1:s. We denote these sets by $B_{1 j}, j=1, \ldots, \nu$. Each of these sets of permutations have $\left(\begin{array}{c}w k+\nu-(w+1) \\ k-(w+1)\end{array}\right)$ members. The sets are not disjoint, for example permutations which have all 1:s in at least two of the 
sets appear in two sets of permutations. Analogously, we have $\left(\begin{array}{c}\nu \\ 2\end{array}\right)$ sets of permutations, the $B_{2 j}: \mathrm{s}, j=1, \ldots,\left(\begin{array}{c}\nu \\ 2\end{array}\right)$, which have at least two sets full of 1 :s. These sets have cardinality $\left(\begin{array}{c}w k+\nu-2(w+1) \\ k-2(w+1)\end{array}\right)$. Observe that each $B_{2 j}$ is the intersection of two $B_{1 j}$ :s.

In general, there are $\left(\begin{array}{c}\nu \\ i\end{array}\right)$ permutation sets $B_{i j}: \mathrm{s}$, having at least $i$ sets of all 1:s, each of which have $\left(\begin{array}{c}w k+\nu-i(w+1) \\ k-i(w+1)\end{array}\right)$ members. Each $B_{i j}$ is the intersection of $i B_{1, j}: \mathrm{s}$. Now recall that if $A_{i}$ are $n$ finite sets, we have the relation

$$
\left|\cup_{i=1}^{n} A_{i}\right|=\sum_{i=1}^{n}(-1)^{i+1}\left(\sum_{\text {all sets } I \subset\{1,2, \ldots, n\} \text { with }|I|=i}\left|\cap_{j \in I} A_{j}\right|\right) .
$$

This generalizes the rule $|A \cup B|=|A|+|B|-|A \cap B|$ for finite sets $A$ and $B$.

By applying the relation it follows that the number of permutations which have all $1: \mathrm{s}$ in at least one set of size $w+1$ is

$$
\sum_{i=1}^{\nu}(-1)^{i+1}\left(\begin{array}{c}
\nu \\
i
\end{array}\right)\left(\begin{array}{c}
w k+\nu-i(w+1) \\
k-i(w+1)
\end{array}\right) .
$$

Next we divide by $\left(\begin{array}{c}w k+\nu \\ k\end{array}\right)$ and let $k \rightarrow \infty$. What remains of the sum is then

$$
\sum_{i=1}^{\nu}(-1)^{i+1}\left(\begin{array}{c}
\nu \\
i
\end{array}\right) w^{-i(w+1)}=1-\left(1-w^{-(w+1)}\right)^{\nu} .
$$

The main part of the estimates in (3) now follow from the weighted sum. By letting $\nu \rightarrow \infty$ in the limit applied on $n=(w-1) k+\nu$, it follows that the limit on $n=w k+\nu$ is bounded from below by $w$, and (3) follows.

(4): One consequence of the theorem is that $g$ is bounded on every straight line $n=w k+\nu, n \geq 1, k \geq 1, w$ and $\nu$ integers. Of course $g(n, k)$ nevertheless is unbounded which follows from the existence of the plateaus, or by taking $w$ large in the estimate (3).

The essence of (3) is that for large enough $\nu$, the limit along the straight line $n=w k+\nu$, is very close to $w+1$. Further, we get from the crude estimate that $g(n, k) \leq w+1$ if $n<k(w+1)$. The increasing property of $g(n, k)$ as a function of $n$ now establishes the fact that the graph of the function $g(n, k)$ contains an infinite series of plataeus, one for each positive integer. For each integer $w+1$, if $\nu$ and $\mu$ are large enough, the values of 
$g$ at the points $(n, k)$ fullfilling $w k+\nu<n, k>\mu$ and $(w+1) k>n$ are in maximum norm arbitrarily close to $w+1$. However, for large $w \nu$ may have to be chosen very large, since $1-w^{-(w+1)}$ is then very close to 1 .

It follows that in certain directions, at the plateaus, the crude estimate is actually very close to the optimal. In neighbourhoods of the straight lines $n=k w$ the estimates differ more: for large $k$ by at most $1+\epsilon$, where $\epsilon$ is small.

Considering $n=k^{2}, w=k$, the weights to $k$ and 1 are $k /\left(\begin{array}{c}k^{2} \\ k\end{array}\right)$ and $k^{k} /\left(\begin{array}{c}k^{2} \\ k\end{array}\right)$ respectively. Since the crude estimate has corresponding weights 1 and 0 , the crude estimate here can be expected to be very far from the optimal.

The references [6] and [7] contain various articles on 0,1-matrices.

\section{References:}

[1] R. A. Brualdi, A. J. Hoffman, On the spectral radius of $(0,1)$-matrices, Linear Algebra and its Applications, 65, (1985) p. 113-146.

[2] R. A. Brualdi, M. Katz, An extremal problem concerning matrices of 0 's and 1's, Linear and Multilinear Algebra, 20, no. 4, (1987) p. 325-331.

[3] R. Krahl, Allocation Problems in Distributed Computer Systems, Inform. Inf. Rep. no. 12, (1990) p. 101-115.

[4] M. Naderi, Modelling and Performance Evaluation of Multiprocessor Organisation with Shared Memories, Comput. Archit. News, vol. 16, no. 4, (Sept 1988) p. 51-74.

[5] C.C Price, Task Allocation in Data Flow Multiprocessors: an annotated bibliography, Comput. Archit. News, vol. 19, no. 1, (March 1991) p. 128-134.

[6] Proceedings of the Twentieth Southeastern Conference on Combinatorics, Graph theory and Computing, Boca Raton, FL (1989).

[7] Proceedings of the Twenty-second Southeastern Conference on Combinatorics, Graph theory and Computing, Baton Rouge, LA, (1991).

[8] J. Rost, A Distributed Algorithm for Dynamic Task Scheduling, CONPAR 90-VAPP, N. Joint Int. Conf. on Vector and Parallel Processing, 
Proceedings, Zurich, Switzerland 10-13 Sept 1990, Springer Verlag, p. 628-639.

[9] B. Shirazi, Ming-fang Wang, Heuristic Functions for Static Task Allocation, Microprocess. Microprogr. (Netherlands) Vol 26, no. 3, (Oct 1989) p. $187-194$.

[10] J. Zahorjan, C. McCann, Processor Sheduling in Shared Memory Multiprocessors, Performance Evaluation Review (USA), vol. 18, no. 1, (May 1990). 


\section{Figure 1. The optimal worst case function $g(n, k)$}

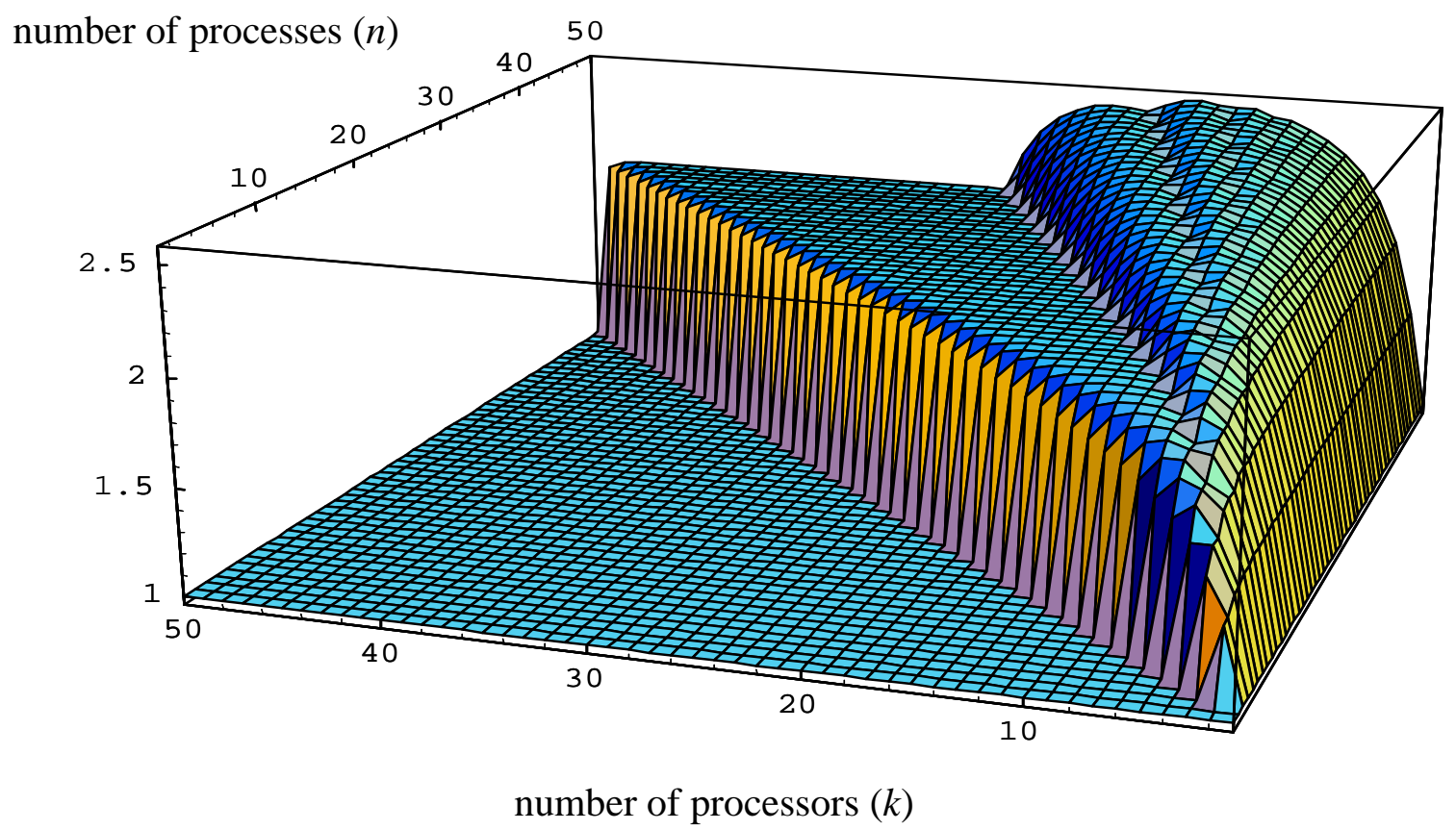

The function $g(n, k)$ is defined as the worst case ratio of static versus dynamic allocations:

$$
g(n, k)=\max _{P} \frac{T_{s}(P)}{T_{d}(P)} .
$$

Here $T_{s}(P)$ and $T_{d}(P)$ denote the execution time for a parallel program $P$ with optimal static and optimal dynamic allocations, respectively, executed on a multiprocessor with $k$ identical processors. The maximum is taken over all parallel programs $\mathrm{P}$ consisting of $n$ processes. 


\section{Figure 2. Ridges and valleys of $\mathbf{g}(\mathbf{n}, \mathrm{k})$}

The ridge/valley structure of the surface $g(n, k)$ is shown here in detail by studying the function $g(n, k)$ as a function of $k$, for each constant $n$. The locations of global maximas ( $)$, local maximas $(\boldsymbol{\square}, \mathbf{B})$ and local minimas ( $\mathbf{⿴ 囗 十 )}$ ) are plotted.
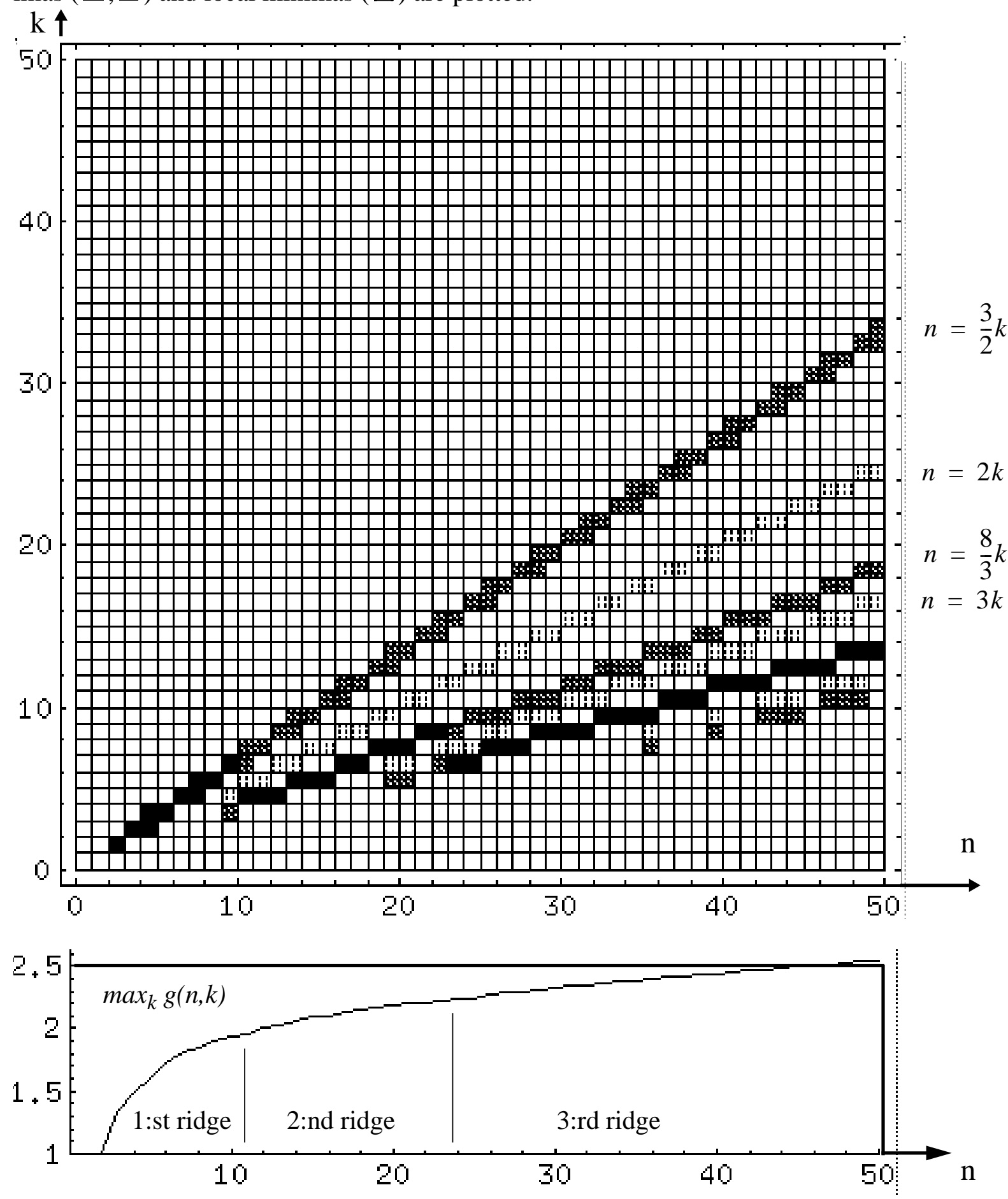

Notes: $1 . g(n, k)$ is increasing as a function of $n$.

2. The straight line equations in the margin are simply constructed from the graph.

3. The function $\max _{k} g(n, k)$ gives the worst case ratio for all parallel programs with $n$ processes and for all multiprocessors with identical processors. 


\section{Figure 3. Bounds on diagonal limit functions}

Functions giving upper and lower bounds for the diagonal limit functions

$$
D(w, v)=\lim _{k \rightarrow \infty} g(w k+v, k)
$$

are plotted, given by the estimates

$$
\max \left(w, w+1-w\left(1-w^{-w-1}\right)^{v}\right) \leq D(w, v) \leq w+1-\left(1-w^{-w-1}\right)^{v} .
$$
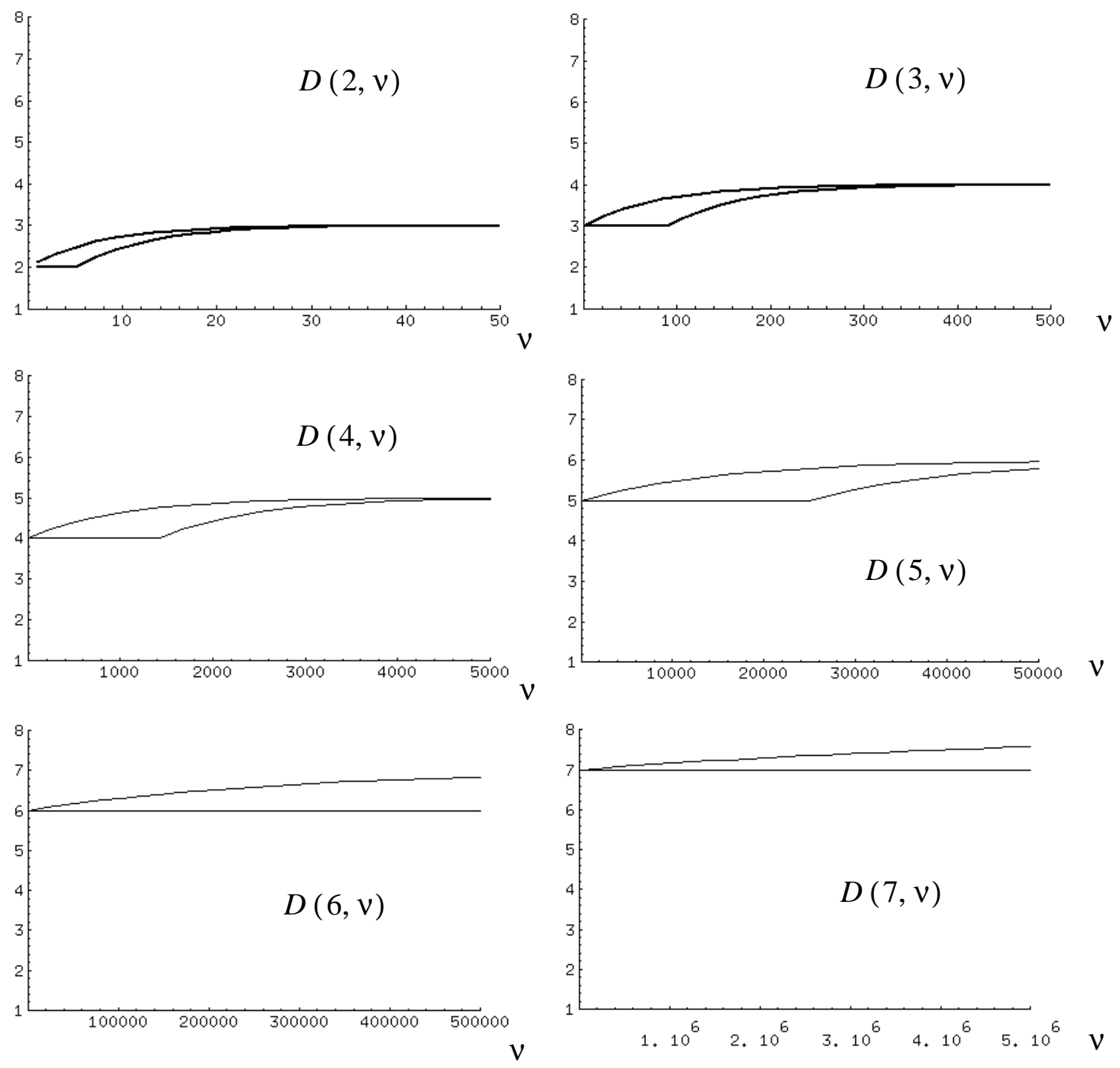

Notes: 1 . Observe the increasing scale on the $v$-axis.

2. The plots illustrate the fact that, at least for large $\mathrm{k}$, the distance from the $w$ :th plateau to the next increases very rapidly with $w$. 